PSFC/JA-98-11

\title{
Neutral Diffusion and Anomalous Effects on Ion Flow Shear
}

\author{
T. Fülöp, Peter J. Catto, P. Helander
}

April 1998

\author{
Plasma Science and Fusion Center \\ Massachusetts Institute of Technology \\ Cambridge, Massachusetts 02139 USA
}

This work was supported by the US Department of Energy, Grant No. DE-FG0291ER54109. Reproduction, translation, publication, use, and disposal, in whole or in part, by or for the US Government is permitted.

Submitted for publication in: Physics of Plasmas 


\title{
Neutral diffusion and anomalous effects on ion flow shear
}

\author{
T. Fülöp ${ }^{(a)}$, Peter J. Catto ${ }^{(b)}$ and P. Helander ${ }^{(c)}$ \\ (a) Department of Elcctromagnetics, Chalmers University of Technology, \\ S-412 96 Götcborg, Sweden \\ (b) Plasma Scicnce and Fusion Ccntcr, Massachusctts Institutc of Technology \\ and Lodcstar Rcscarch Corporation, Cambridgc, MA 02139 \\ (c) UKAEA Fusion, Culham Scicncc Centrc (UKAEA/Euratom Association), \\ Abingdon, Oxon, OX14 3DB, Unitcd Kingdom
}

\begin{abstract}
Bulk ion flow can be modificd by ion-ncutral intcractions in the cdgc region of tokamaks where ncutral atoms arc abundant. In this region, the standard ncoclassical expression for the ion flow is not consistent with the experimental obscrvations. Previous work in the platcau regime [Valanju, ct al., Phys Fluids B 4, 2675 (1992)] suggests that the discrepancy is causcd mainly by momentum loss duc to ion-ncutral charge cxchangc. The present work shows that in the abscnce of ncutral diffusion, charge cxchange cannot affect the ncoclassical transport of the ions significantly if the ncutral mean-frec path is short and the neutral density is low compared to the plasma density. However, the inclusion of ncutral diffusion and anomalous processes in the platcau regime can substantially modify parallel ion flow inside the scparatrix as detcrmined by the flux surface averaged momentum constraint. Morcover, the local shcar in the flow may be determined by a balance betwecn neutral diffusion and anomalous cffects which may then modify the turbulent transport.
\end{abstract}

\footnotetext{
${ }^{\perp}$ Email: tunde@elmagn.chalmers.se
} 


\section{Introduction}

The influence of the neutral atoms on the ion parallel flow has been of interest recently, since the discrepancy between experimental measurements, [1], and neoclassical predictions of the ion flow are in some cases correlated with neutral concentration.

Earlier work, [2,3], suggested that charge exchange (CX) processes have an important role in modifying the ion flow in the edge plasma, where the plasma temperature and density are lower than in the core and the neutral concentration is high. However, the analysis of Ref.[2] is not consistent with its neutral short mean-free path assumption, since it assumes stationary neutrals. Reference [3] used the neoclassical moment approach including the effect of CX processes with stationary neutrals and made quantitative comparisons with experiment to show that neutrals have a dramatic effect on the main ion poloidal rotation. However, the assumption that the parallel neutral flow $V_{\| n} \simeq 0$ is inappropriate for edge plasma where the neutral penetration depth and CX mean-free path for a neutral are on the order of a centimeter. The assumption of stationary neutrals made in Refs. $[2,3]$ cannot be made without also including anomalous diffusion. The neutrals will only have vanishing mean parallel velocity if they have not yet collided with any ions. In that case they are moving into the plasma, which in steady state requires a balancing efflux of ions. Moreover, we will show that without the neutral diffusion, CX processes would not affect the transport of ions significantly.

The purpose of this work is to derive an ion drift kinetic equation that includes the effect of neutral diffusion due to CX. Lsing the resulting drift kinetic equation we 
demonstrate how the $\mathrm{CX}$ process and anomalous transport modify the parallel and poloidal ion flow, assuming short mean-free path neutrals and plateau ions. The ability of anomalous transport to influence parallel dynamics has been noted previously, [4]. The sharp radial (and perhaps poloidal) variation of the neutrals and anomalous transport cause them to introduce a strong shear into the poloidal flow and thereby the electrostatic potential. Consequently, the neutrals and anomalous processes may influence the sheared flow stabilization observed in reduced transport regimes, [5]. By working in the plateau regime, we are relying on a large aspect ratio to make expansions valid. However, this somewhat artificial limit allows us to illustrate the mechanisms at work more simply.

The rest of the paper is organized as follows. In Section II we derive the kinetic equation of the ions, including the effect of neutral and anomalous ion diffusion. In Section III we solve a simplified version of the kinetic equation in the plateau limit and investigate the modification of the parallel ion flow due to the diffusive terms. Section IV is devoted to a discussion of the results. Finally, in Section V we summarize the conclusions.

\section{Derivation of the kinetic equation}

In the neutral kinetic equation we retain charge exchange and ionization so that

$$
v \cdot \nabla f_{n}=X\left(f_{i}, f_{n}\right)-\nu_{z} f_{n},
$$


where $X$ denotes the charge exchange operator and $\nu_{z}=n_{\varepsilon}\langle\sigma v\rangle_{z}$ is the ionization frequency. We then let

$$
f_{n}=\frac{n_{n}}{n_{i}} f_{i}+h
$$

to obtain

$$
v \cdot \nabla\left(\frac{n_{n}}{n_{i}} f_{i}+h\right)=\langle\sigma v\rangle_{x}\left(n_{n} f_{i}-n_{i} f_{n}\right)-\nu_{z} f_{n}=-\left(\nu_{x}+\nu_{z}\right) h-\nu_{z} \frac{n_{n}}{n_{i}} f_{i},
$$

where $\nu_{x}=n_{i}\langle\sigma v\rangle_{x}$ is the $\mathrm{CX}$ frequency for neutrals. Note that this definition of the CX collision frequency differs by a factor $n_{n} / n_{i}$ from the one used in Ref.[1-3] where $\nu_{x}$ referred to the ions. The short mean-free path solution $\left(v_{l i} \ll W \nu_{x}\right)$ to Eq.(1) valid for $\nu_{x}>\nu_{z}$ is

$$
h=-\frac{1}{\nu_{x}+\nu_{z}} \nabla \cdot\left(\frac{n_{n}}{n_{i}} v f_{i}\right)-\frac{\nu_{z}}{\nu_{x}+\nu_{z}} \frac{n_{n}}{n_{i}} f_{i}+\ldots
$$

where $v_{l i}^{2}=2 T_{i} / m_{i}$ and $W$ is the local equilibrium scale length associated with the edge in the presence of neutrals. Inserting $h$ into the sum of the ion and neutral kinetic equations

$$
\boldsymbol{v} \cdot \nabla\left(f_{i}+f_{n}\right)+\boldsymbol{a} \cdot \nabla_{v} f_{i}-C\left(f_{i}\right)=0
$$

where $\boldsymbol{a}=(Z e / M)[\boldsymbol{E}+(\boldsymbol{v} \times \boldsymbol{B}) / c]$ and $C$ is the ion-ion collision operator, gives

$$
\boldsymbol{v} \cdot \nabla\left[\left(1+\frac{\nu_{x}}{\nu_{x}+\nu_{z}} \frac{n_{n}}{n_{i}}\right) f_{i}\right]+\boldsymbol{a} \cdot \nabla_{v} f_{i}-C\left(f_{i}\right)=\nabla \cdot\left[\boldsymbol{v} \frac{1}{\nu_{x}+\nu_{z}} \nabla \cdot\left(\frac{n_{n}}{n_{i}} \boldsymbol{v} f_{i}\right)\right]
$$

where the right hand side represents neutral diffusion. Ionization appears diffusive because it is occurring in the presence of $\mathrm{CX}$ which causes the neutrals to respond diffusively, but it is important to remember that Eq.(6) is only valid for $\nu_{x} \gtrsim \nu_{z}$.

Writing

$$
f_{i}=\bar{f}_{i}+\tilde{f}_{i}
$$


where $\bar{f}_{i}=<f_{i}>_{\varphi}$ is the gyroaveraged part of the ion distribution function and $\tilde{f}_{i}=$ $f_{i}-\bar{f}_{i}$ is the gyrophase dependent part, and assuming $\Omega \gg \nu_{i i}$, with $\Omega=Z e B / M c$, and $|\boldsymbol{E}| \ll|(\boldsymbol{v} \times \boldsymbol{B}) / \boldsymbol{c}|$, the gyrophase dependent term is given by

$$
\tilde{f}_{i}=\frac{1}{\Omega} v \times b \cdot \nabla\left[(1+\eta) \bar{f}_{i}\right]
$$

where $\boldsymbol{b}=\boldsymbol{B} / \boldsymbol{B}$ and we have introduced $\eta=\nu_{x} n_{n} / n_{i}\left(\nu_{x}+\nu_{z}\right)$. Inserting Eq.(8) and gyroaveraging Eq.(6) yields the sum equation

$$
\begin{array}{r}
v_{\|} \nabla_{\|}\left[(1+\eta) \bar{f}_{i}\right]+(1+\eta) v_{d} \cdot \nabla\left[(1+\eta) \bar{f}_{i}\right]+(1+\eta)\left\langle\left\langle\delta v_{E} \cdot \nabla \delta f_{i}\right\rangle_{\varphi}\right\rangle_{f l u c l} \\
-C\left(\bar{f}_{i}\right)=\nabla\left[\frac{1}{\nu_{x}+\nu_{z}} \nabla:\left(\frac{n_{n}}{n_{i}}\langle v v\rangle_{\varphi} \bar{f}_{i}\right)\right] \equiv U \bar{f}_{i}
\end{array}
$$

where $U$ is the neutral diffusion operator, $\langle v v\rangle_{\varphi}=v_{\perp}{ }^{2} / 2(I-b b)+v_{\|}^{2} b b$, and $v_{d}=$ $\left[v_{\perp}{ }^{2} /(2 \Omega)\right] b \times \nabla \ln B+\left(v_{\|}^{2} / \Omega\right) \boldsymbol{b} \times(\boldsymbol{b} \cdot \nabla \boldsymbol{b})$. We retain anomalous transport to balance neutral diffusion by introducing the fluctuating $\boldsymbol{E} \times \boldsymbol{B}$ drift $\delta \boldsymbol{v}_{E}$ and the fluctuating portion of the ion distribution function $\delta f_{i}$ with $\langle\ldots\rangle_{\text {fluct }}$ denoting an average over turbulent fluctuations. If we note that $\delta f_{i} \sim \tau_{c} \delta v_{E} \cdot \nabla \delta \bar{f}_{i}$ with $\tau_{c}$ the correlation time for the fluctuations, then

$$
D_{a n} \tilde{f}_{i} \equiv-\left\langle\left\langle\delta \boldsymbol{v}_{E} \cdot \nabla \delta f_{i}\right\rangle_{\varphi}\right\rangle_{f l u c l} \sim \frac{\partial}{\partial r}\left(D \frac{\partial \bar{f}_{i}}{\partial r}\right)
$$

where $D$ is the anomalous diffusivity and we assume electrostatic fluctuations.

Changing to $v$ and $\xi=v_{\|} / v$ variables gives

$$
v_{\|} \nabla_{\|}=\frac{v_{\|}}{q R} \frac{\partial}{\partial \theta}-\left(1-\xi^{2}\right) \frac{\epsilon v_{\|}}{2 q R} \sin \theta \frac{\partial}{\partial \xi}=\omega \frac{\partial}{\partial \theta}+M
$$

where $\epsilon=r / R, \theta$ is the poloidal angle, $r$ and $R$ the minor and major radii, and $M$ is the magnetic mirror term. In the new variables, the gyroaveraged sum equation with 
the $\eta \sim n_{n} / n_{i} \ll 1$ terms neglected becomes

$$
\omega \frac{\partial g}{\partial \theta}+M g-C g=-v_{d} \cdot \nabla f_{M}-M f_{d}+U \bar{f}_{i}+D_{a n} \bar{f}_{i}
$$

where $\omega=v_{\|} / q R, C$ is the linearized collision operator, and we have let

$$
\bar{f}_{i}=f_{M}+f_{d}+g=f_{M}+\frac{2 V_{\| i} v \xi}{v_{l i}^{2}} f_{M}+g
$$

with $V_{\| i}$ a lowest order flux function,

$$
f_{M}=n_{i}\left(\frac{m_{i}}{2 \pi T_{i}}\right)^{3 / 2} \exp (-(E-e \Phi) / T)
$$

and $E=M v^{2} / 2+e \Phi$.

\section{Solution and flows in the plateau regime}

To lowest order we may solve for $g$ by neglecting $M g$ and $\left(U+D_{a n}\right) \bar{f}_{i}$. In the plateau regime, $\epsilon \ll 1$ allows us to neglect $M g$. Then using $V_{\| i} / v_{l i} \sim q \rho_{i} / \epsilon W \ll 1$ in $M f_{d}$, gives $g / f_{M} \sim q \rho_{i} / W \ll 1$. Note that the assumption $V_{\| i} / v_{l i} \sim q \rho_{i} / \epsilon W$ can be verified a posteriori. The neglect of neutral and anomalous diffusion to lowest order corresponds to $v_{d} \cdot \nabla f_{M} \gg\left(U+D_{a n}\right) f_{M}$ which for $D_{u n} \sim U$ requires $n_{n} / n_{i} \ll$ $W \nu_{x} / R \Omega$. To next order we keep all the terms in Eq.(12) to evaluate the flux surface averaged parallel momentum constraint which gives the parallel ion flow as modified by neutral and anomalous diffusion.

Making the usual plateau replacement $C f=-\nu f$, the lowest order version of Eq. (12) becomes

$$
\omega \frac{\partial g}{\partial \theta}+\nu g=\frac{v^{2}\left(1+\xi^{2}\right)}{2 \Omega R} \frac{\partial f_{M}}{\partial r} \sin \theta+\frac{\epsilon}{2} \frac{\omega}{\xi} \sin \theta\left(1-\xi^{2}\right) \frac{\partial f_{d}}{\partial \xi}
$$


where

$$
g=\frac{(\nu \sin \theta-\omega \cos \theta) Q}{\nu^{2}+\omega^{2}} f_{M}
$$

and

$$
Q=\frac{v^{2}}{2 \Omega R}\left\{\left(1+\xi^{2}\right)\left[\frac{p_{i}^{\prime}}{p_{i}}+\frac{e \Phi^{\prime}}{T_{i}}+\left(\frac{v^{2}}{v_{l i}^{2}}-\frac{5}{2}\right) \frac{T_{i}^{\prime}}{T_{i}}\right]+\left(1-\xi^{2}\right) \frac{2 \Omega r}{q R} \frac{V_{\| i}}{v_{l i}^{2}}\right\}
$$

We use a prime to denote a radial derivative. Equations (15)-(17) are the same as in Ref.[2].

To obtain the parallel ion flow, in the plateau regime, we return to $\mathrm{Eq} .(12)$, take the $v_{\|}$moment, and perform a flux surface average. Most of the terms in Eq.(12) will vanish, leaving only terms assumed to be higher order, namely

$$
\left\langle\int v \xi M g d^{3} v\right\rangle=\left\langle\int v \xi\left(C^{+}+D_{a n}\right) f_{d} d^{3} v\right\rangle
$$

where $\langle\ldots\rangle$ denotes flux surface averaging. This equation determines the parallel ion flow, $V_{\| i}$, and represents a balance between the damping of the parallel flow on the left and the accumulation of momentum due to diffusion on the right. Evaluating the different terms in Eq.(18) gives

$$
\begin{aligned}
\frac{\sqrt{\pi}}{4} \epsilon \frac{n_{i} v_{l i}^{3}}{\Omega R}\left(\frac{p_{i}^{\prime}}{p_{i}}+\frac{e \Phi^{\prime}}{T_{i}}+\frac{T_{i}^{\prime}}{2 T_{i}}+\frac{2 \Omega \epsilon}{q} \frac{V_{\| i}}{v_{l i}^{2}}\right)= & \frac{1}{2} \frac{\partial}{\partial r}\left[\frac{1}{\nu_{x}+\nu_{z}} \frac{\partial\left(\left\langle n_{n}\right\rangle_{\theta} v_{l i}^{2} V_{\| i}\right\rangle}{\partial r}\right]+ \\
& +\left\langle\left\langle\left\langle\delta v_{E} \cdot \nabla \int d^{3} v v_{\|} \delta f_{i}\right\rangle_{\varphi}\right\rangle_{f l u c t}\right\rangle_{\theta}
\end{aligned}
$$

where $\langle\ldots\rangle_{\theta}$ denotes a poloidal average, i.e. $\left\langle n_{n}\right\rangle_{\theta}=(2 \pi)^{-1} \oint d \theta n_{n}$. In the absence of neutrals and anomalous processes $\mathrm{Eq}$.(19) reduces to the usual plateau result.

The modification due to neutrals and anomalous processes can be estimated by writing the parallel ion flow schematically as

$$
V_{\| i}=G U_{n e o}
$$


where the usual plateau result is

$$
U_{n c o}=-\frac{T}{m_{i} \Omega} \frac{q}{\epsilon}\left(\frac{p_{i}^{\prime}}{p_{i}}+\frac{e \Phi^{\prime}}{T_{i}}+\frac{T_{i}^{\prime}}{2 T_{i}}\right)
$$

Using Eq.(10) to crudely estimate the anomalous term as

$$
\left\langle\left\langle\left\langle\delta v_{E} \cdot \nabla\left(\tau_{c} \delta v_{E} \cdot \nabla n_{i} V_{\| i}\right)\right\rangle_{\varphi}\right\rangle_{j l u c l}\right\rangle_{\theta} \sim \frac{\partial}{\partial r}\left(D \frac{\partial n_{i} V_{\| i}}{\partial r}\right)
$$

gives

$$
G^{-1} \sim 1+O\left[\frac{q R}{v_{l i} \epsilon^{2}}\left(\frac{n_{n}}{n_{i}} \frac{v_{l i} \lambda}{W^{2}}+\frac{D}{W^{2}}\right)\right]
$$

In the expression for $G, \lambda=v_{i i} /\left(\nu_{x}+\nu_{z}\right)$ is the mean-free path and $\left(n_{n} V_{\| i}\right)^{\prime \prime} /\left(n_{n} V_{\| i}\right) \sim$ $W^{-2} \sim\left(n_{n} V_{\| i} v_{l i}^{2}\right)^{\prime \prime} /\left(n_{n} V_{\| i} v_{l i}^{2}\right)$. Lsing the preceding, the poloidal flow may be written as

$$
V_{p i}=\left(c / e B n_{i}\right)\left[(1-G)\left(p_{i}^{\prime}+e n_{i} \Phi^{\prime}\right)-G n_{i} T_{i}^{\prime} / 2\right]
$$

Consequently, the neutral diffusion and anomalous processes may introduce strong shear in the poloidal flow.

To see whether substantial changes are possible we make comparisons between the various terms in the flux surface averaged momentum constraint, using the following set of parameters: safety factor $q=3$, inverse aspect ratio $\epsilon=1 / 3$, the neutral to ion density ratio $n_{n} / n_{i}=10^{-3}$, the ion thermal velocity $v_{l i}=10^{7} \mathrm{~cm} / \mathrm{s}$, the characteristic radial scale length $W=2 \mathrm{~cm}$, major radius $R=1 \mathrm{~m}$, mean-free path $\lambda=1 \mathrm{~cm}$ and $D=1 \mathrm{~m}^{2} / \mathrm{s}$. Comparing the magnitude of the mirror term with the neutral diffusion term

$$
\frac{\left\langle\int v \xi U f_{d} d^{3} v\right\rangle}{\left\langle\int v \xi M g d^{3} v\right\rangle} \sim \epsilon^{-2} \frac{n_{n}}{n_{i}} \frac{q R \lambda}{W^{2}} \sim 1
$$


so that these terms are comparable. Thus, within the limitations of a plateau ordering, taking the neutral diffusion term the same order as the mirror term is justified at the edge, just inside the separatrix. Comparing this anomalous diffusion term with the neutral diffusion term we obtain

$$
\frac{\left\langle\int v \xi D_{a n} f_{d} d^{3} v\right\rangle}{\left\langle\int v \xi U f_{d} d^{3} v\right\rangle} \sim \frac{n_{i}}{n_{n}} \frac{D}{v_{l i} \lambda} \sim 1
$$

Therefore, the two terms are comparable and the contributions from both should be included in the ion parallel flow. That both appear should not be surprising since in the steady state the anomalous outward diffusion of ions is balanced by an influx of recycled neutrals.

To verify that our orderings are consistent we note that Eq.(18) implies that $M g \sim U_{d} f_{d}$ while the neglect of $U^{*}$ in our lowest order equation (15) requires $\omega \partial g / \partial \theta \gg$ $U f_{M}$. As a result, we need $V_{\| i} / \epsilon v_{l i} \sim q \rho /\left(\epsilon^{2} W\right) \gg 1$ which allows us to satisfy our assumption

$$
1 \gg \frac{n_{n}}{n_{i}} \frac{\Omega R}{\nu_{x} W} \sim\left(\frac{n_{n}}{n_{i}} \frac{q R \lambda}{\epsilon^{2} W^{2}}\right)\left(\frac{\epsilon^{2} W}{q \rho_{i}}\right) .
$$

We need to ensure the magnetic field satisfies $q \rho_{i} / W \ll 1$. Thus for the parameters listed $B \sim 10 \mathrm{kG}$ is required. The reason such a low $B$ is needed can be traced back to the limitations of the plateau model which requires $\epsilon \ll 1$ while we have taken $\epsilon=1 / 3$.

\section{Discussion}

In Ref.[2], the ion kinetic equation is employed with ionization neglected. Therefore $v \cdot \nabla f_{i}+a \cdot \nabla_{v} f_{i}=C\left(f_{i}\right)-X\left(f_{i}, f_{n}\right)$ is considered. In the gyrophase averaged equation 
for $g$, the replacement

$$
X\left(f_{i}, f_{n}\right) \rightarrow X\left(f_{M}+f_{d}, \frac{n_{n}}{n_{i}} f_{M}\right)=\nu_{x} \frac{n_{n}}{n_{i}} f_{d}
$$

is employed, which corresponds to setting $V_{\| n}=0$. However, from parallel neutral momentum conservation $M n_{n} \nu_{x}\left(V_{\| i}-V_{\| n}\right)=\nabla_{\|} p_{n}$ so a consistent treatment would have given

$$
X\left(f_{i}, f_{n}\right) \rightarrow \nu_{x} \frac{n_{n}}{n_{i}}\left(f_{d i}-f_{d n}\right)=\nu_{x} \frac{n_{n}}{n_{i}} f_{M} \frac{M v_{\|}}{T}\left(V_{\| i}-V_{\| n}\right)
$$

which would have only yielded terms small in $n_{n} / n_{i} \ll 1$ in the short mean-free path limit. In other words, since $V_{\| i} \simeq V_{\| n}$ in the short mean-free path limit, the Galilean invariance of the CX operator ensures negligible corrections unless diffusive terms are retained.

Analogously, if $V_{\| i} \simeq V_{\| n}$, the parallel ion flow will be unaffected also by the elastic ion-neutral collisions, to lowest order. This follows from the Galilean invariance property of the collision operator giving $C_{i n}\left(f_{M i}, f_{d n}\right)+C_{n i}\left(f_{d i}, f_{M n}\right) \simeq 0$, which means that these terms will not contribute to the flux surface averaged momentum constraint. This argument is independent of the form of the collision operator and can be used for both the CX and elastic collision operators. In addition, the elastic ion-neutral collision operator will give a diffusive contribution to the parallel ion flow, just as the CX operator does. However, the analysis of the elastic operator is considerably more difficult, since it is a complicated Boltzmann operator. Terms qualitatively similar to those obtained from $\mathrm{CX}$ interactions between the neutrals and ions are anticipated. 


\section{Concluding remarks}

The main conclusion of this work is that the neutral diffusion and anomalous processes affect the parallel and poloidal ion flows significantly. This observation is of importance for understanding the discrepancies between experimental observations and neoclassical predictions of the flows. It also means that the lowest order expression $\left(Z e n_{i} / M\right)\left(\boldsymbol{E}+c^{-1} \boldsymbol{v} \times \boldsymbol{B}\right)=\nabla\left(p_{i}+p_{n}\right)$ is not automatically valid for edge plasmas since neutral diffusion and anomalous processes can be substantial.

The neutral density variation on the scale length of the penetration depth $\left(l_{p} \simeq\right.$ $v_{t i} / \sqrt{\nu_{x} \nu_{z}}$ ) introduces a strong shear into the parallel ion flow. Anomalous processes may also cause $\left\langle\left\langle\left\langle\delta v_{E} \cdot \nabla \int d^{\beta} v v_{\|} \delta f_{i}\right\rangle_{\varphi}\right\rangle_{\text {slucl }}\right\rangle_{\theta}$ to have strong radial variation. As a result, the amount of shear in the parallel and poloidal ion flow just inside the separatrix may be determined by a delicate balance between the effects of neutral diffusion and anomalous processes which may thereby play a role in the suppression of turbulent transport and the $\mathrm{L}$ to $\mathrm{H}$ mode transition.

\section{Acknowledgments}

We would like to thank Sergei Krasheninnikov for enlightening discussions and T.F. is inclebted to the Plasma Science and Fusion Center at Massachusetts Institute of Technology for its hospitality. This work was supported jointly by L'.S. Department of Energy grant DE-FG02-91ER-54109, by the European Community under an association contract between Euratom and Sweden and by the L.K. Department of Trade and Industry and Euratom. 


\section{References}

[1] W. L. Rowan, A. G. Meigs, E. R. Solano, P. M. Valanju, M. D. Calvin, and R. D. Hazeltine, Phys. Fluids B 5, 2485, (1993)

[2] P. M. Valanju, M. D. Calvin, R. D. Hazeltine, and E. R. Solano, Phys. Fluids B $4,2675,(1992)$

[3] P. Monier-Garbet, K. H. Burrell, F. L. Hinton, J. Kim, X. Garbet, and R. J. Groebner, Nucl. Fusion, 37, 403, (1997)

[4] P. Helander, R. D. Hazeltine, and P. J. Catto, Phys. Rev. Lett. 77, 2479, (1996)

[5] K. H. Burrell, Phys. Plasmas, 4, 1499, (1997) 